\title{
Primary spontaneous complete colporrhexis
}

\author{
P S Wijesinghe'
}

(Index words: Post-partum haemmorhage, management)

Spontaneous rupture or avulsion of the vagina from the cervix during labour is a rare condition. The term colporrhexis describes this obstetric injury and it is subdivided into primary or secondary, spontaneous or traumatic, complete or incomplete. Incomplete colporrhexis includes rupture of the vaginal epithelium and the muscularis, whereas complete involves the overlying peritoneum as well. Primary colporrhexis is a vaginal vault tear not associated with cervical or uterine extension. Secondary colporrhexis cannot be differentiated from a rupture that has originated in the uterus and then extended to involve the vagina (1).

The condition has been associated with high parity, but it has been reported in primigravidae (2). Most cases of primary colporrhexis are of traumatic origin associated with unskilled instrumental delivery. The aetiology of the rarer primary spontaneous colporrhexis is unknown and previous vaginal trauma has been implicated. Precipitate labour and use of oxytocics in labour are other factors Jescribed $(1,3)$. A misdirection of the uterine axis due to a pendulous abdomen leading to marked anteversion of the uterus. ventrofixation of the uterus, evacuation of a full rectum after an enema, and prolapse leading to altered blood supply to the vagina are the other factors responsible. I describe here a case of primary spontaneous complete colporthexis.

\section{Case report}

A 27-year old woman was seen in her second preganancy. At a twin delivery two years earlier, she had gone into preterm labour at 35 weeks of amenorrhoea and undergone internal podalic version to correct transverse lie of the second twin.

The antenatal period of her second pregnancy, which was a singleton pregnancy with a cephalic presentation, was uneventful. She went into spontaneous labour at 39 weeks and delivered a healthy baby with a birth weight of $2.9 \mathrm{~kg}$. The placenta was delivered by active management of the third stage of labour. During the immediate postpartum period she continued to bleed vaginally with evidence of hypovolaemic shock. The suprapubic area was tender. Vaginal examination showed a tear involving the upper anterior vagina, and a haematoma was felt in the vesico-uterine pouch. Uterine rupture involving the cervix and the vagina was suspected and a laparotomy was done. At laparotomy the uterus was found to be intact. There was a haematoma underneath the bladder peritoneum and the vagina was found to have avulsed completely from the cervix anteriorly and the anterior lip of the cervix was visible through the deficit. The posterior vaginal wall was intact. The vault of the vagina was sutured on to the cervix in two layers. She was sterilised at the same time on her request. She made an uneventful recovery.

\section{Discussion}

Although the clinical picture in this patient was that of a rupture of the uterus, during surgery it turned out that the uterus was intact and spontaneous avulsion of the vagina from the cervix had taken place. The internal podalic version done in her first pregnancy to deliver the second of twin could have damaged and weakened the attachment of the vagina to the cervix making it vulnerable. However, the injury occurred without any instrumentation and involved the peritoneum overlying the ruptured vagina. This fits the description of primary spontaneous complete colporrhexis.

Four methods of treatment have been described. The first is to allow healing by secondary intention, which is now obsolete. The second involves two-layer closure, and the third is to close in one layer. The fourth option is to perform a hysterectomy when the exposure is poor, and when there is severe blood loss. Although primary spontaneous complete colporrhexis is rare, the possibility should be considered in a patient who develops vaginal bleeding and hypovolaemic shock in labour or in the immediate post-partum period.

\section{References}

1. Brander JH, Buchman MI. Rupture of the vagina during spontaneous delivery. Obstetrics and Gynecology 1964; 24: 151-4.

2. Sharma KKY, Frith KM. Primary complete colporthexis in a primigravida. British Journal of Obstetrics and Gynaecology 1993; 100: 1142-44.

3. Osterling DL, McCreedy PA, McFee JG, Thompson HE. Primary complete colporrhexis. Obstetrics and Gynecology 1971; 38: 96-8. 